\title{
CAVITY FIELDS APPROACH TO SIMPLE PSEUDOSPIN MODEL OF ORIENTATIONAL GLASS
}

\author{
L. De Cesare ${ }^{a}$, K. Lukierska-Walasek ${ }^{b}$, I. Rabuffo ${ }^{a}$ and K. Walasek ${ }^{b}$ \\ ${ }^{a}$ Dipartimento di Fisica Teorica e sue Metodologie per le Scienze Applicate \\ Università degli Studi di Salerno, 84100 Salerno, Italy \\ ${ }^{b}$ Institute of Physics, Pedagogical University of Zielona Góra \\ Plac Słowiański 6, 65-069 Zielona Góra, Poland
}

\begin{abstract}
A simple infinite-range model of axial quadrupolar glass is investigated within the cavity-ficlds approach inside a pure state and a cluster of pure states. Working at a level of a pure state, the nonlinear susceptibility is derived. When a cluster of pure states is considered, all the known results of the one-step replica-symmetry breaking approach are easily reproduced. Besides, the nonlinear susceptibility and the stability conditions are obtained and related numerical results are presented. In this way the stability range of the replica-symmetry breaking solution, quite difficult to be derived within the replica method, is established on the purely physical ground. An interesting feature is that, at any considered stage, the nonlinear susceptibility diverges at a given temperature $T_{\mathrm{c}}$ where the quadrupolarization and the quadrupolar glass order parameters are nonzero and finite. This may be interpreted as a signal of a glassy phase transition not in the Landau sense.
\end{abstract}

PACS numbers: 05.20.-y, 64.60.Cn, 64.70.Pf, 75.10.Nr, 75.50.Lk

In recent years there has been a significant growth of interest in quadrupolar glasses (QGs) or more general orientational glasses for which intensive experimental and theoretical studies [1-8] have been achieved, from the pioneering paper by Sullivan et al. [3], in close analogy to SGs. The term "quadrupolar glass" refers to the low-temperature phase of molecular crystals with randomly frozen orientations of the quadrupolar bearing molecules. The simplest example of a molecular crystal possessing orientational disorder is a solid orthohydrogen-parahydrogen mixture $\left(o-p-\mathrm{H}_{2}\right)$, with a concentration of $o-\mathrm{II}_{2}$ species lower than $55 \%$, in which orthomolecules with rotational momentum equal to unity are dissolved in a matrix of crystalline parahydrogen, whose molecules have zero angular momentum and spherical symmetry. Other well-known systems are $o-p-\mathrm{D}_{2}$ crystals with low concentration of $p-\mathrm{D}_{2}$ molecules, Ar diluted with $\mathrm{N}_{2}$ molecules and $(\mathrm{KBr})_{1-x}(\mathrm{KCN})_{x^{-}}$ type mixed crystals $[1,2]$. Although the presence of randomness and frustration makes these materials similar to SGs, the absence of reflection symmetry [2] gives 
rise to deviations from the conventional SG scheme and to a peculiar behaviour of QGs.

From a theoretical point of view, there has not been yet a convincing theory for these more complex glassy systems. In general, a study of QG properties, based on realistic microscopic Hamiltonians, is a rather complicated task due to the complexity of the involved interactions and it is very difficult to treat it more deeply along this direction. Rather, it is certainly desirable and convenient to investigate simplest models capturing the essential physics of real disordered systems. A simple model along this line was proposed by Lutchinskaia et al. [4] for $o-p-\mathrm{H}_{2} \mathrm{QG}$ in the spirit of the famous model of Sherrington and Kirkpatrick (SK) [9] for SGs. The model, describing the glassy properties of a system of $N$ quadrupoles with a strong anisotropy in $z$-direction, is defined by the IIamiltonian

$$
H=-\frac{1}{2} \sum_{i, j=1}^{N} J_{i j} Q_{i} Q_{j} .
$$

Ifere, $J_{i j}$ 's are quenched random interactions between quadrupoles located at sites $i$ and $j, Q_{i}=\left(S_{i}^{z}\right)^{2}-2$ is the $z z$-component of the molecular quadrupole second-rank tensor and $S_{i}^{z}=0, \pm 1$ is the $z$-component of the angular momentum (pseudospin) of the molecule at site $i$. With the model (1) it is assumed that each quadrupole in the system is oriented along the distinguished $z$-axis. In solid $o-p-\mathrm{IH}_{2}$ mixtures with a concentration $\alpha<0.55$ of $o-\mathrm{H}_{2}$, nuclear magnetic resonance (NMR) experiments at a very low temperature [3] show evidence that the local $z$-axes are just randomly oriented. In the following, we find convenient to choose [10] the quadrupolar couplings as random variable taking two possible values $J_{i j}= \pm J / \sqrt{N}$ (we will assume $J \equiv 1$ ) with the equal probability for \pm signs.

The SK-like replica symmetric (RS) solution of the model (1) was obtained in Ref. [4], where it was shown that the model exhibits a mixed phase with the global quadrupolar orientation and QG order parameter increasing continuously with decreasing temperature. The absence of the trivial solution for these order parameters suggests that the glass regime is established gradually with decreasing temperature without a phase transition in the Landau sense. This result, although obtained on the ground of a simple model, appears to be in agreement with experimental data [3]. The stability analysis [5] of RS solution shows that it becomes unstable below a well-defined temperature $T_{\mathrm{c}} \approx 1.367 \mathrm{~J} / k_{\mathrm{B}}\left(k_{\mathrm{B}}\right.$ is the Boltzmann constant). Quite recently [6], the one-step of the replica-symmetry breaking (RSB) using the Parisi ansatz [10] has been performed in a wide range of temperature including $T=0$. However a stability study of the RSB solution is prevented by intrinsic mathematical difficulties of the replica formalism.

A new method, named "the cavity-fields approach" has been developed [10] for SK model. This approach seems rather promising towards a proper description of SG systems. It avoids the replica trick, relies on well-known mathematics and deals with quantities having a transparent physical meaning, such as local magnetizations and random magnetic fields. In view of the limited space available for the present paper we only briefly report the main results of a systematic study of the axial QG model (1) using the cavity-fields approach (for more explanations 
see Ref. [8]). We work inside a pure state and inside a cluster of pure states [10] taking into account also the correlation eflects for large but finite $N$.

According to the main idea of the cavity-fields approach, we add a new quadrupole $Q_{0}$ at site 0 , so that the model (1) moves from $N$ to $(N+1)$ quadrupole with Hamiltonian $H_{N+1}=H_{N}-Q_{0} \sum_{i=1}^{N} J_{0 i} Q_{i}$, where $J_{0 i}= \pm 1 / \sqrt{N}$ is the random variable with the equal probability for \pm signs. The field

$$
h_{0}=\sum_{i=1}^{N} J_{0 i} Q_{i}
$$

produced by a given configuration of $N$ quadrupoles and acting on the site 0 once the corresponding new quadrupole has been removed, is called the cavity field.

Proceeding in a strict analogy with the method described in Ref. [10] we get, at the level of a pure state, the results equivalent to the RS treatment [4]. The calculation of nonlinear susceptibility, at the level of a pure state, gives the limit of stability RS solution the same as that obtained in Ref. [5] by the De Almeida-Thouless method [11]. Working within the cluster of pure states one obtains the equations for the QG parameters $q_{1}$ and $q_{0}$, global quadrupolarization $m$ and breakpoint $x$ of the QG parameter Parisi function [10]. The results coincide with those given in Ref. [6]. Within the cavity field approach $q_{1}$ and $q_{0}$ are physically interpreted as the Edwards-Anderson parameter for QG, i.e. $q_{1}=(1 / N) \sum_{1}^{N}\left(m_{i}^{a}\right)^{2}$ and the interstate overlap, i.e. $q_{0}=(1 / N) \sum_{1}^{N} m_{i}^{a} m_{i}^{b}$, where $m_{i}^{a}$ and $m_{i}^{b}$ denotes local quadrupolarizations in pure states $a$ and $b$, respectively, which belong to the cluster of states [10].

A stability condition of the RSB solutions [6] can be easily formulated, within the cavity-fields method, by calculation of the nonlinear susceptibility $\chi_{\mathrm{nl}}$ for the cluster of states [8]. The result for $\chi_{\mathrm{nl}}$ is

$$
\chi_{\mathrm{nl}}=\beta^{-2} \frac{(1-X)^{2}}{X}
$$

where

$$
X=1-342 \beta^{2} \int_{-\infty}^{\infty} \frac{\mathrm{d} z}{\sqrt{2 \pi}} \mathrm{e}^{-z^{2} / 2}\left[\frac{\int_{-\infty}^{\infty} \frac{\mathrm{d} y}{\sqrt{2 \pi}} \mathrm{e}^{-y^{2} / 2} Z_{0}^{x} \mathrm{e}^{6 \theta} /\left(2 \mathrm{e}^{6 \theta}+1\right)^{4}}{\int_{-\infty}^{\infty} \frac{\mathrm{d} y}{\sqrt{2 \pi}} \mathrm{e}^{-y^{2} / 2} Z_{0}^{x}}\right] \geq 0
$$

with $Z_{0}=2 \mathrm{e}^{\theta}+\mathrm{e}^{-2 \theta}$ and $\theta=\beta\left(y \sqrt{q_{1}-q_{0}}+z \sqrt{q_{0}}\right)-\frac{\beta^{2}}{2}\left(2-m-q_{1}\right)$. Using the numerical results for parameters $m, q_{1}, q_{0}$ and $x$, already known from a previous study [6], from the condition $\chi_{\mathrm{nl}}^{-1} \geq 0$ or $X \geq 0$, we firstly find that $X$ vanishes exactly at the temperature $T_{\mathrm{c}}=1.367 \mathrm{~J} / k_{\mathrm{B}}$, where the ergodicity is broken. Besides, in the region $T<T_{\mathrm{c}}$, the positivity of $X$ is found to occur in the interval of temperature $T_{\mathrm{c}}^{*}<T<T_{\mathrm{c}}$ with $T_{\mathrm{c}}^{*} \approx 0.637 T_{\mathrm{c}}$. In this region the RSB solution is stable. At $T=T_{c}^{*}$, the stability is broken again $(X<0$ as $\left.T<T_{c}^{*}\right)$. In Fig. 1 the temperature dependence ( $T$ dependence) of the quantity $X(4)$, i.e. the denominator of the nonlinear susceptibility for a cluster of states is plotted. For a comparison the $T$ dependence of $X$ obtained inside a pure state is also given. In the region of instability $T<T_{\mathrm{c}}^{*}$, the RSB solution (see Fig. 1) show a clear tendency towards the stability stronger than RS solution. Of course, below the second temperature $T_{c}^{*}$ of the stability breaking, the QG model (1) 


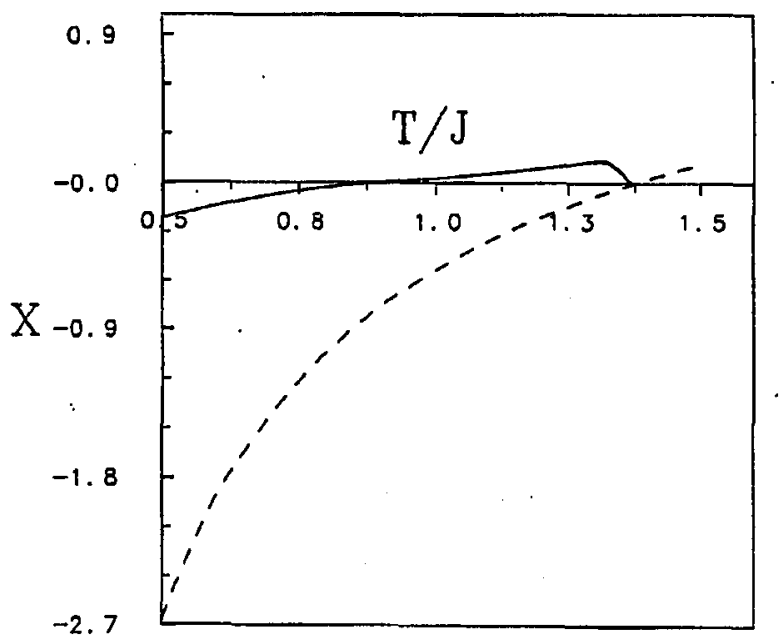

Fig. 1. Plot of the nonlinear susceptibility denominator $X$ as a function of the reduced temperature $k_{\mathrm{B}} T / J$ according to the present predictions of the cavity-fields approach (the solid line). The dashed line refers to the RS solution, stable for $T \geq T_{\mathrm{c}}$ and unstable for $T<T_{\mathrm{c}}$, with $T_{\mathrm{c}} \approx 1.367 \mathrm{~J} / k_{\mathrm{B}}$.

should be investigate within the next stages of the cavity approach (a level of superclusters [10]). In the light of the present numerical results, we expect that the window of stability below $T_{\mathrm{c}}$ will enlarge at any next stage.

Two of us (K.L.-W. and K.W.) would like to express their appreciation for the warm hospitality of the Department of Theoretical Physics of Salerno University shown to them during the preparation of this paper. Additional support from the Committee for Scientific Research, grant No. 2 P302 264 03, is gratefully acknowledged.

\section{Reforences}

[1] U.T. Höchli, K. Knorr, A. Loidl, Adv. Phys. 39, 405 (1990).

[2] K. Binder, J.D. Reger, Adv. Phys. 41, 547 (1992) and references therein.

[3] N.S. Sullivan, M. Devoret, P.B. Cowan, C. Urbina, Phys. Rev. B 17, 5016 (1978); N.S. Sullivan, Can. J. Chem. 66, 908 (1988).

[4] E.A. Lutchinskaia, V.N. Ryzhov, E.E. Tareyeva, J. Phys. C17, L665 (1984); Theor. Math. Phys. 67, 623 (1986).

[5] K. Walasek, Phys. Rev. B 51, 9314 (1995).

[6] K. Walasek, K. Lukierska-Walasek, M. Wodawski, Phys. Rev. $B$ (in press).

[7] E.A. Lutchinskaia, E.E. Tareyeva, Phys. Rev. B 52, 366 (1995).

[8] L. De Cesare, K. Lukierska-Walasek, I. Rabufo, K. Walasek, unpublished.

[9] D. Sherrington, S. Kirkpatrick, Phys. Rev. Lett. 32, 1972 (1975).

[10] M. Mezard, G. Parisi, M.A. Virasoro, Spin Glass Theory and beyond, in series World Scientific Lecture Notes in Physics, Vol. 9, World Scientific, Singapore 1987.

[11] J.R.L. De Almeida, D.J. Thouless, J. Phys. A 11, 983 (1978). 